\title{
Regulatory Effect of Resveratrol and Prednisolone on MDR1 Protein Expression in Acute Lymphoblastic Leukemia Cell Line (CCRF-CEM)
}

\author{
Mehdi Talebi ${ }^{1}$, Sina Bahar Aghdam², Ako Azimi ${ }^{3}$, Hamed Mohammadi², \\ Somayyeh Karimi Yonjali ${ }^{4}$, Maryam Asariha ${ }^{5,6}$, Milad Zadi Heydarabad ${ }^{7 *}$
}

\begin{abstract}
Objective: Chemotherapy is the most widely recognized technique to regard leukemia and also different sorts of human tumors. In any case, tranquilize protection has stayed as the primary test against the adequacy of medications. Besides, having different unfriendly impacts, chemotherapy drugs are getting to be traded by characteristic modalities for growth treatment. In such manner, natural segments, for example, resveratrol and prednisolone have been recognized to sharpen the leukemic cells to modified cell demise through an arrangement of complex procedures. In this investigation, we have analyzed effect of 15,50 and $100 \mu \mathrm{M}$ of resveratrol and $700 \mu \mathrm{M}$ of prednisolone on the human multidrug protection quality 1 (MDR1) as a notable marker for cell sedate protection. We assessed the impact of resveratrol and prednisolone on MDR1 protein expression in the CCRF-CEM cell line as an agent for intense lymphoblastic leukemia. The investigation was planned to clear up whether. Materials and methods: CCRF-CEM cells linage get under drug treatment with use of resveratrol and prednisolone. Western blot use at 24 and 48 hours with different doses of resveratrol and prednisolone to analysis of MDR1 expression changes. Results: Effect of 15, 50, and 100 micro molar of resveratrol and 700 micro molars of prednisolone on CCRF-CEM cells led to the MDR1 decrease. Western blot use for evaluation of MDR1 protein expression changes. Conclusion: In the present study, we observed that resveratrol and prednisolone, with a dose-dependent effect, can reduce the expression of the MDR1 protein. This reduction of expression demonstrates that resveratrol and prednisolone can overcome to drug resistance created by MDR1.
\end{abstract}

Keywords: Protein expression- acute lymphoblastic leukemia- resveratrol- prednisolone- MDR1 gene

Asian Pac J Cancer Prev, 20 (4), 1171-1176

\section{Introduction}

An obtrusive and quickly developing growth of White Blood Cells (WBC) is the Acute Lymphoblastic Leukemia (ALL) which generally starts from bone marrow (Azimi et al., 2015; Azimi et al., 2016; Ahani-Nahayati et al., 2018). ALL is presently considered as the most common threat in youngsters, containing $77 \%$ of leukemic cases in this age gathering which is most every now and again happened in offspring of 2-5 years of age (Stanulla and Schrappe, 2009; Azimi et al., 2015; Azimi et al., 2016; Zadi Heydarabad et al., 2018). Since the revelation of ALL, few lines of medication specialists have centered to create compelling and safe modalities to keep the development and movement of this threat. An extensive variety of cures were planned with different sorts of systems; however, their danger and antagonistic impacts have been reliably remained the principle hindrance in front of productive treatment. Concerns about the danger and wide range of reactions, these days expanding interests are concentrated to supplant the concoction specialists by more regular or plant-based parts to treat the human diseases (Zhang et al., 2010; Rauf et al., 2017).

Resveratrol (trans-3,5,4' -trihydroxystilbene), a constituent of red wine, has been appeared to have against oxidative properties which is found in excess of 70 types of plants, for example, pines, shelled nut, mulberry and grape(Gusman et al., 2001; Azimi et al., 2015; Azimi et al., 2016). Pathogen contamination, condition changes, daylight, overwhelming metals, and ozone presentation are a few sorts of stresses that incite resveratrol creation in plants (Athar et al., 2007). Previous examinations have demonstrated that the resveratrol has numerous valuable impacts from chemoprevention of tumors to hostile to oxidative, against aggravation, cardio assurance and immunomodulation, in any case the correct

${ }^{1}$ Hematology and Oncology Research Center, ${ }^{2}$ Immunology Research Center, ${ }^{4}$ Stem Cell Research Center, Tabriz University of Medical Sciences, Tabriz, ${ }^{3}$ Department of Basic Sciences, Maragheh University of Medical Sciences, Maragheh, ${ }^{5}$ Medical Biology Research Center, ${ }^{6}$ Student Research Committee, Kermanshah University of Medical Sciences, Kermanshah, ${ }^{7}$ Medicinal Plants Research Center, Yasuj University of Medical sciences, Yasuj, Iran.*For Correspondence: milad.zadi88@gmail.com 
atomic component is yet to be cleared up (Dong, 2003). Resveratrol has been shown to be prepared to do avoiding tumor angiogenesis and metastasis and stifles three noteworthy phases of tumor extension including tumor start, tumor advancing, and advancement. The utilization of resveratrol to treat the medication protection for chemoprevention has been archived in light of in vitro and in vivo discoveries (Seve et al., 2005). a few examinations on human cells have demonstrated that different pathways engaged with cell development, cell passing and irritation are managed by resveratrol(Seve et al., 2005). Various examinations from in vitro tries have likewise checked that resveratrol can smother the multiplication of an assorted scope of cells (Aggarwal et al., 2004). Broad examinations have upheld additionally the part of glucocorticoids (GCs, for example, prednisolone in a few cell forms including digestion, separation, and expansion and cell survival. Especially, in lymphoid framework such operators impact the cell cycle, and influence immunoglobulin and cytokine creation, and initiate apoptosis in youthful lymphoblast. GC impacts on invulnerable framework are for the most part required amid the age of the insusceptible collection and in addition the immunomodulation (Schaaf and Cidlowski, 2002; Azimi et al., 2016). In this way these specialists are legitimate contender to be used in the treatment of youth intense lymphoblastic leukemia (ALL) and different malignancies influencing lymphoid framework (Azimi et al., 2016). Among the distinctive instruments by which the malignancy cells may ensure their survival, expansion, and intrusion the MDR-1 has been considered as a key potential giver amid tumor movement. The MDR1 quality encodes the medication transporter P glycoprotein (Pgp) which advances medicate efflux. The most broadly thinks about system by which the human disease what's more, tumor cell lines oppose to chemotherapy is the over-articulation of Pgp. Different components of medication efflux are intervened by transporter proteins including the multidrug resistance associated protein (MRP) (Zaman et al., 1994) and over-articulation of the significant vault protein (LRP) (Scheffer et al., 1995). Changes in topoisomerase compounds and a modified glutathione/ glutathione -S-transferase (GSH/GST) detoxification framework (Hao et al., 1994) are likewise speak to instruments of cell protection from cytotoxic specialists.

We have also examined the effect of resveratrol and prednisolone on the gene expression and methylation level of DNA promoters of the MDR1 gene in our previous study and observed that resveratrol and prednisone cause decrease in MDR1 gene expression without altering the methylation level of the MDR1 gene promoter (Zadi Heydarabad et al., 2018).

In another study that was done by Al-Abd et al., (2011) resveratrol inhibited P-gp and reduced the expression of MDR1 in cancer cell lines in vitro. Gautam et al., (2000) illustrated some effects of proliferative inhibition of resveratrol on leukemic cell lines (U937, HL-60) during a series of laboratory studies.

We have also examined the effect of resveratrol and prednisolone on the gene expression and methylation level of DNA promoters of the MDR1 gene in our previous study and observed that resveratrol and prednisone cause decrease in MDR1 gene expression without altering the methylation level of the MDR1 gene promoter (Zadi Heydarabad et al., 2018).

Due to the low toxicity of resveratrol in vivo as well as in vitro and its anti-leukemic effects, being selective of its effects and be useful as an anti-leukemic agent; We decided to assess the effect of resveratrol and prednisolone on overcoming drug resistance caused by increased expression and function of MDR1 by changing its expression and reducing its expression in CCRF-CEM cancer cells.

\section{Materials and Methods}

\section{Cell Culture}

As a harmful cell line speaking to ALL, the CCRF_CEM cell line was bought from Pasteur organization of Tehran, Iran. The cells were refined routinely in RPMI 1640 medium supplemented with $10 \%$ warmth inactivated fetal cow-like serum (GIBCO, USA), streptomycin (10mg/ml) and $10,000 \mathrm{U} / \mathrm{mL}$ of penicillin. The cells were kept up at $37^{\circ} \mathrm{C}$ with $5 \% \mathrm{CO}_{2}$. Preceding resveratrol treatment the cells were seeded in $60 \mathrm{~mm}$ culture dishes at a thickness of $4 \times 10^{5}$ cells for each dish.

\section{Treatment}

Resveratrol (98\% immaculateness, Sigma Aldrich, Germany) was broken up in ethanol what's more, added to culture dishes at the convergences of 15,50 and $100 \mu \mathrm{M}$. Following 24 and 48 hours, the cells were gathered for protein extraction. Prednisolone (98\% immaculateness, Sigma-Aldrich, Germany) was broken up in DMSO and added to CCRF-CEM societies at the centralization of 700 $\mu \mathrm{M}$ (Azimi et al., 2015; Azimi et al., 2016) At last, the cells were collected after $24 \mathrm{~h}$ and $48 \mathrm{~h}$ for RNA extraction.

\section{Assessment of MDR1 protein}

In order to assess the alteration of protein (MDR1) expression, the Western Blot technique used. Hence, $5 \times 10^{6}$ cells were cultured in $25 \mathrm{~cm}^{2}$ flasks in the final volume of $5 \mathrm{ml}$. Subsequently, the cells treated with 15, 50 and $100 \mu \mathrm{M}$ of resveratrol and $700 \mu \mathrm{M}$ of prednisolone for 24 and 48 hours.

\section{Extraction and isolation of proteins}

The cells were harvested and washed by cold phosphate buffer saline. Equivalent amounts of cell lysis buffer, including 1\% NP-40, 0.5\% SDS, 10mM Tris-HCL $(\mathrm{pH}=7.4), 5 \mathrm{mM}$ EDTA, $150 \mathrm{mM} \mathrm{NaCl}, 100 \mu \mathrm{M}$ PMSF, $0.5 \%$ sodium deoxycholate, and protease-phosphatase inhibitor were added to cells. 13000RPM was considered to centrifuge samples for $20 \mathrm{~min}$ in $-4^{\circ} \mathrm{C}$. Then, the supernatant discarded, and Bradford assay used for evaluation of protein concentration. According to Laemmli assay, the equivalent amounts of protein samples were separated using 10\% SDS-PAGE.

\section{Western Blot}

Transference of bands from gel to nitrocellulose membrane fulfilled Using Mini Trans-Blot Bio-Rad. 


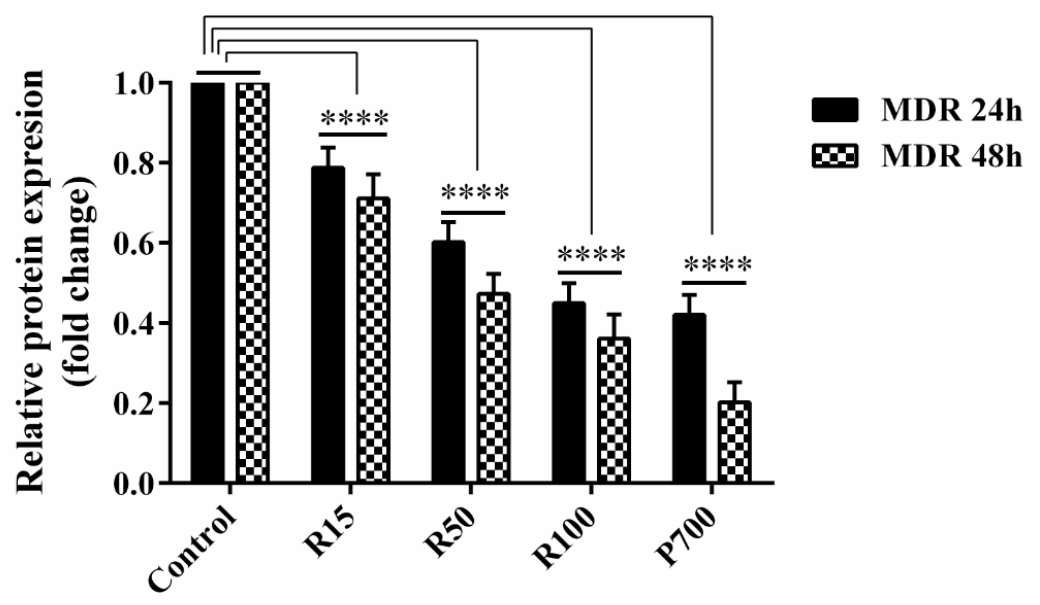

Figure 1. The Effects of Resveratrol and Prednisolone on MDR1 Protein Expression in CCRF-CEM Cells Compared to Control Cells, Cultured with FBS, at 24 and 48 hours after Treatment.

After that, the membrane was washed and exposed with blocking solution, containing 5\% skim milk in TBS-T, for 1 hour. Subsequently, the cells were washed with TBS-T and exposed to the primary monoclonal antibody in the presence of blocking solution at $-4^{\circ} \mathrm{C}$ for a whole night. Anti-actin antibody was considered as an internal control. Then, the membrane was washed in three steps by TBS-T, exposed with secondary antibody, conjugated with HRP, at room temperature for 1 hour. Eventually, chemiluminescence assay was used to the visualization of protein bands.

The effect of resveratrol and prednisolone on CCRF-CEM cells was significant and reduced MDR1 expression. The Western blot method was applied to the measurement semi quantitatively of MDR1 protein expression (Figure 2).

In order to assess changes in MDR1 protein expression, the western blot technique was used. The Actin protein considered as an internal control. Western blot results illustrated decline in MDR1 expression influenced by 15, 50 and $100 \mu \mathrm{M}$ of resveratrol and $700 \mu \mathrm{M}$ of Prednisolone for 24 and 48 hours (Figure 1). As shown in Figure 1 and Figure 2, the treatment of CCRF-CEM cells with 15, 50 and $100 \mu \mathrm{M}$ of resveratrol and $700 \mu \mathrm{M}$ of prednisolone for 24 and 48 hours illustrated a significant decrease of MDR1 expression based on the results obtained from densitometry analysis of MDR1 protein bands.

Additionally, the western blot technique was used to P-gp (a protein encoded by MDR1) quantitative measurement.

\section{Statistically Analysis}

In order to do densitometry and assess the bands, the Image J software and data analysis carried out by SPSS 20.0 software were used. The immunoblotting and flow cytometry data were obtained from three distinct experiments and demonstrated as a \pm mean standard error. For studying the differences between groups, paired T-test was used and the $p$ values $<0.05$ were considered as statistically significant.

\section{Results}

Resveratrol and prednisolone decrease the expression of MDRl gene without altering the level of methylation of DNA promoters

In our previous study, to investigate the effect of resveratrol and prednisolone on the level of methylation of MDR1 DNA gene promoters and their effects on MDR1 gene expression in acute lymphoblastic cell CCRF-CEM cells, we measured these cells at doses of 15, 50 and $100 \mu \mathrm{m}$ of resveratrol and $700 \mu \mathrm{m}$ of prednisolone and treated them for 24 and 48 hours. Our results in that study showed that resveratrol and prednisone cause decrease in MDR1 gene expression without altering MDR1 gene methylation pattern in a dose and time-dependant manner (Zadi Heydarabad et al., 2018).

Resveratrol and prednisolone cause decrease in MDR1 protein expression

As indicated by the past looks into, the convergences of resveratrol required for apoptosis enlistment in the CCRFCEM cells were 15,50 and $100 \mu \mathrm{M}$, while $700 \mu \mathrm{M}$ of prednisolone appeared to be ideal. Likewise, Resveratrol and prednisolone cause a diminishing in MDR1 quality articulation in a period and focus subordinate way.

MDR 24h

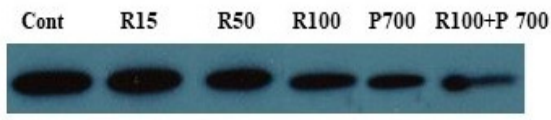

MDR 48h

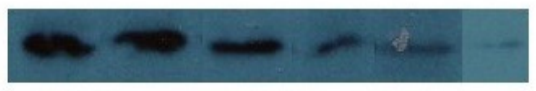

\section{$\beta$-Actin}

Figure 2. The MDR1 protein level. Results are representative of the reduction in MDR1 at doses of 15, 50 and $100 \mu \mathrm{M}$ of Resveratrol and $700 \mu \mathrm{M}$ of Prednisolone after treatment, as compared to the protein level in control group (untreated cells). The band intensity of MDR1 was quantitated and normalized with b-actin band and expressed as relative MDR1 expression. 
Following 24 and 48 hours of treatment, the varieties of expression levels of MDR1 protein were assessed contrasted and untreated control cells. To look at the adjustments in expression levels of MDR1 protein, the CCRF-CEM cells were treated with resveratrol and prednisolone. It was watched that resveratrol and prednisolone have significant effect on expression levels of MDR1 protein CCRF-CEM cells in the measurements and interims utilized as a part of this investigation. Our result demonstrated that resveratrol in the groupings of 15,50 and $100 \mu \mathrm{M}$ of Resveratrol and prednisolone $700 \mu \mathrm{M}$ can diminish MDR1 quality articulation (Figure 1 and Figure 2) following 24 and 48 hours of treatment. Having a noteworthy down-direction of MDR1 quality articulation following resveratrol treatment in different diseases (Azimi et al., 2015) we were intrigued to examine the expression levels of MDR1 protein after treatment by resveratrol and in addition prednisolone in various measurements and treatment interims. To play out this, Western blot method was utilized to demonstrate the changes of expression levels of MDR1 protein in these cells. As appeared in Figure 1, we observed changes of expression levels of MDR1 protein following resveratrol and prednisolone treatment taking all things together assigned dosages and interims have Resveratrol and prednisolone cause a diminishing in MDR1 protein expression.

Moreover, Western blot analysis also showed a significant decrease in MDR1 expression in CCRF-CEM cells treatment with concentration of 15,50 and $100 \mu \mathrm{M}$ of Resveratrol and $700 \mu \mathrm{M}$ of Prednisolone after 24 and 48 hours (Figure 2).

Results are representative of the reduction in MDR1 at doses of 15,50 and $100 \mu \mathrm{M}$ of Resveratrol and $700 \mu \mathrm{M}$ of Prednisolone after treatment, as compared to the protein level in control group (untreated cells). The band intensity of MDR1 was quantitated and normalized with b-actin band and expressed as relative MDR1 expression.

\section{Discussion}

These days, the chemotherapy, surgery and radiotherapy are the most widely recognized methodologies for treatment of tumor. Considering drug protection, one of the normal strategies to battle malignancies is to sharpen the threatening cells to restorative operators (Gupta et al., 2011; Pouyafar et al., 2018). In spite of the fact that a number of novel obtrusive restorative systems are accessible, a difficult issue in hostile to leukemia treatments is protection from apoptosis which is in any event to some degree a reason of treatment protection. Consequently, apoptosis acceptance is a key component to bring wanted medication proficiency.

Reducing the expression of MDR1 gene can be effective in decreasing the effects of drug resistance, improving the chemotherapy outcome and rescuing patients who are resistant to treatment from the effects of severe toxic chemotherapy (Robert and Jarry, 2003). MDR1 and MRP1 are members of the large ABC family of protein transductors; it is believed that they are an undesirable prognostic factor in the response to and survival of malignancies (Ahani-Nahayati et al., 2018; (Den Boer et al., 1999). In the event of a successful use of natural materials to reduce the effect of drug resistance, we will face some minor side effects. Resveratrol is one of the natural ingredients associated with known anti-cancer effects. These effects associated with the reduction of the expression and function of $\mathrm{ABC}$ carriers which reflects a significant reduction in IC50 medications.

Resveratrol is a natural part that has an assortment of organic impacts on malignant cells counting against proliferative, calming and chemo preventive impacts (Cecchinato et al., 2007; Rauf et al., 2018; Zadi Heydarabad et al., 2018). It is cleared up that resveratrol can make the safe harmful cells delicate to treatment through a few systems. It expands the declaration of CD95L and passing receptors interceded apoptosis (Azimi et al., 2015), changes NF-KB pathway and incites customized cell passing(Estrov et al., 2003; Azimi et al., 2016), changes STAT3 articulation by diminishing the capacity of tyrosine kinase Src and directs the survivin acceptance (target quality of STAT3), and fortifies the p53 task and NO generation(Gogada et al., 2011), Besides, GCs act by means of a ligand initiated translation factor known as GC receptor In a wide range of natural examples, the assessment of changes of protein level is a particular procedure for concentrate of expression levels of qualities. In spite of being non-quantitative, western blot is a valuable procedure especially as a confirmation test for prove changes of expression of a protein by two different drugs or substances.

Considering the above-mentioned items, we investigated whether resveratrol and prednisolone can reduce the expression of MDR1 in the level of protein as a strong barrier against anticancer drugs and one of the main causes of drug resistance, as well as overcome the drug resistance, increasing the expression and function of the MDR1 protein by its reducing (Amin, 2013; Zadi Heydarabad et al., 2018; Ghasemi et al., 2018; Hano et al., 2018). In the present study, our results show that resveratrol and prednisolone, with a dose-dependent effect, can reduce the expression of the MDR1 protein. Reducing the expression of the MDR1 illustrates that resveratrol and prednisolone can overcome the drug resistance by MDR1. In our previous research, we demonstrated that resveratrol and prednisolone can reduce the expression of MDR1 gene without changing the methylation pattern of the MDR1 gene (Zadi Heydarabad, et al., 2018). Our observation on these acute lymphoblastic cells CCRF-CEM also showed that resveratrol and prednisolone can increase the apoptosis of these cancer cells by changing the expression of microRNAs miR 15a and miR16-1 and also reducing the expression of BAX gene as well as increasing expression of BCL-2 without changing the pattern of methylation of the promoter of BAX and BCL-2 genes (Azimi et al., 2015; Azimi et al., 2016; Heydarabad et al., 2016; Zadi Heydarabad et al., 2018; Zadi Heydarabad et al., 2018). In the present study, we also found that resveratrol and prednisolone can overcome drug resistance caused by increasing the expression of MDR1 in acute lymphoblastic cell CCRF-CEM via reducing the expression of MDR1 in 
the protein level. The issue of overcoming drug resistance has always been an important factor in the elimination of cancer resistant cells to cure. In other studies, such as a study carried out by Quan F, resveratrol reduced the expression of MDR1 (P gp gene) and Bcl 2 (antiapoptotic) in chemotherapy-resistant epidermoid cell line, KB 200 (Quan et al., 2008). In another study that was done by Al-Abd et al., (2011) resveratrol inhibited P-gp and reduced the expression of MDR1 in cancer cell lines in vitro. Gautam et al., (2000) illustrated some effects of proliferative inhibition of resveratrol on leukemic cell lines (U937, HL-60) during a series of laboratory studies.

Previous studies have shown that resveratrol causes decrease in MDR1 gene expression by inhibiting NF-kB activation as well as the signaling pathway of p38 MAPK (Zhang et al., 2016).

In a study carried out by Vasil F. Chekhun and Galina I. Kulik on the resistance of the human MCF-7 / R breast cancer cell line to doxorubicin, it was concluded that the development of MCF-7 / R drug resistance to doxorubicin, they concluded that the development of MCF-7 / R drug resistance to doxorubicin is the result of a change in the expression of P gp (Chekhun, et al., 2006). Chekhun et al., (2006) reported the relationship between MDR1 promoter methylation and drug resistance in the T-cell line as well as three patients with chronic lymphocytic leukemia. Expression of P-gp is the main barrier in chemotherapy for hematological malignancies, including acute myeloid leukemia (Mahadevan and List, 2004; Chekhun et al., 2006; de Moraes et al., 2013; Gao et al., 2015).

In conclusion, in the present study, ability of resveratrol and prednisolone in reducing the expression of the MDR1, with dose-dependent effect, was observed. Reducing the expression of the MDR1 demonstrates that resveratrol and prednisolone can overcome the drug resistance induced by MDR1.

\section{Disclosures}

The authors state that there is no conflict of interest.

\section{Acknowledgements}

This work was supported by Tabriz University of Medical Sciences [grant numbers 94/15].

\section{References}

Aggarwal BB, Bhardwaj A, Aggarwal RS, et al (2004). Role of resveratrol in prevention and therapy of cancer: preclinical and clinical studies. Anticancer Res, 24, 2783-40.

Ahani-Nahayati M, Solali S, Asanjan KS, et al (2018). Promoter methylation status of survival-related genes in MOLT-4 cells co-cultured with bone marrow mesenchymal stem cells under hypoxic conditions. Cell J (Yakhteh), 20, 188.

Al-Abd A, Mahmoud A, El-Sherbiny G, et al (2011). Resveratrol enhances the cytotoxic profile of docetaxel and doxorubicin in solid tumour cell lines in vitro. Cell Proliferat, 44, 591-601

Amin ML (2013). P-glycoprotein inhibition for optimal drug delivery. Drug Target Insights, 7, DTI. S12519.

Athar M, Back JH, Tang X, et al (2007). Resveratrol: a review of preclinical studies for human cancer prevention. Toxicol Appl Pharmacol, 224, 274-83
Azimi A, Hagh M, Yousefi B, et al(2016). The effect of prednisolone on miR 15a and miR16-1 expression levels and apoptosis in acute lymphoblastic leukemia cell line: CCRF-CEM. Drug Res, 66, 432-5.

Azimi A, Hagh MF, Talebi M, et al (2015). Time-and concentration-dependent effects of resveratrol on miR 15a and miR16-1 expression and apoptosis in the CCRF-CEM acute lymphoblastic leukemia cell line. Asian Pac J Cancer Prev, 16, 6463-8.

Cecchinato V, Chiaramonte R, Nizzardo M, et al (2007). Resveratrol-induced apoptosis in human T-cell acute lymphoblastic leukaemia MOLT-4 cells. Biochem Pharmacol, 74, 1568-74.

Chekhun VF, Kulik GI, Yurchenko OV, et al (2006). Role of DNA hypomethylation in the development of the resistance to doxorubicin in human MCF-7 breast adenocarcinoma cells. Cancer Lett, 231, 87-93.

de Moraes ACR, Maranho CK, Rauber GS, Santos-Silva MC (2013). Importance of detecting multidrug resistance proteins in acute leukemia prognosis and therapy. $J$ Clin Lab Anal, 27, 62-71.

Dong Z (2003). Molecular mechanism of the chemopreventive effect of resveratrol. Mutat Res-Fund Mol M, 523, 145-50.

Estrov Z, Shishodia S, Faderl S, et al (2003). Resveratrol blocks interleukin-1 $\beta$-induced activation of the nuclear transcription factor $\mathrm{NF}-\mathrm{\kappa B}$, inhibits proliferation, causes S-phase arrest, and induces apoptosis of acute myeloid leukemia cells. Blood, 102, 987-95.

Gao F, Dong W, Yang W, et al (2015). Expression of P-gp in acute myeloid leukemia and the reversal function of As2 3 on drug resistance. Oncol Lett, 9, 177-82.

Gautam S, Xu Y, Dumaguin M, Janakiraman N, Chapman R (2000). Resveratrol selectively inhibits leukemia cells: a prospective agent for ex vivo bone marrow purging. Bone Marrow Transplant, 25, 639.

Ghasemi A, Khanzadeh T, Zadi Heydarabad M, et al (2018). Evaluation of BAX and BCL-2 gene expression and apoptosis induction in acute lymphoblastic leukemia cell line CCRFCEM after high-dose prednisolone treatment. Asian Pac J Cancer Prev, 19, 2319-23.

Gogada R, Prabhu V, Amadori M, Scott R, Hashmi S, Chandra D (2011). Resveratrol induces p53-independent, X-linked inhibitor of apoptosis protein (XIAP)-mediated Bax protein oligomerization on mitochondria to initiate cytochrome c release and caspase activation. $J$ Biol Chem, 286, 28749-60.

Gupta SC, Kannappan R, Reuter S, Kim JH, Aggarwal BB (2011). Chemosensitization of tumors by resveratrol. Ann N Y Acad Sci, 1215, 150-60.

Gusman J, Malonne H, Atassi G (2001). A reappraisal of the potential chemopreventive and chemotherapeutic properties of resveratrol. Carcinogenesis, 22, 1111-7.

Hano M, Tomášová L, Šereš M, et al (2018). Interplay between p-Glycoprotein expression and resistance to endoplasmic reticulum stressors. Molecules, 23, 337.

Hao X-Y, Bergh J, Brodin O, Heltman U, Mannervik B (1994). Acquired resistance to cisplatin and doxorubicin in a small cell lung cancer cell line is correlated to elevated expression of glutathione-linked detoxification enzymes. Carcinogenesis, 15, 1167-73.

Heydarabad MZ, Vatanmakanian M, Nikasa M, HAGH MF (2016). Epigenetic regulation of specific transcription factors in osteogenic differentiation of mesenchymal stem cells. Turk J Biol, 40, 1040-9.

Huang F, Wu X-N, Chen J, Wang W-X, Lu ZF (2014). Resveratrol reverses multidrug resistance in human breast cancer doxorubicin-resistant cells. Expl Exploratory Ext, 7, 1611-6. 
Mahadevan D, List AF (2004). Targeting the multidrug resistance-1 transporter in AML: molecular regulation and therapeutic strategies. Blood, 104, 1940-51.

Pouyafar A, Heydarabad MZ, Mahboob S, Mokhtarzadeh A, Rahbarghazi R (2018). Angiogenic potential of YKL-40 in the dynamics of tumor niche. Biomed Pharmacother, 100, 478-85.

Quan F, Pan C, Ma Q, Zhang S, Yan L (2008). Reversal effect of resveratrol on multidrug resistance in KBv200 cell line. Biomed Pharmacother, 62, 622-9.

Rauf A, Imran M, Butt MS, et al (2018). Resveratrol as an anti-cancer agent: A review. Crit Rev Food Sci Nutr, 58, 1428-47.

Schaaf MJ, Cidlowski JA (2002). Molecular mechanisms of glucocorticoid action and resistance. J Steroid Biochem Mol Biol, 83, 37-48.

Scheffer GL, Wijngaard PL, Flens MJ, et al (1995). The drug resistance-related protein LRP is the human major vault protein. Nat Med, 1, 578-82.

Seve M, Chimienti F, Devergnas S, et al (2005). Resveratrol enhances UVA-induced DNA damage in HaCaT human keratinocytes. Med Chem, 1, 629-33.

Stanulla M, Schrappe M (2009) Treatment of childhood acute lymphoblastic leukemia. In: Seminars in hematology. Elsevier, pp 52-63.

Zadi Heydarabad M, Baharaghdam S, Azimi A, et al (2019). The role of tumor suppressor of resveratrol and prednisolone by downregulation of YKL-40 expression in CCRF-CEM cell line. J Cell Biochem, 120, 3773-9.

Zadi Heydarabad M, Nikasa M, Vatanmakanian M, Azimi A, Farshdousti Hagh M (2018). Regulatory effect of resveratrol and prednisolone on MDR1 gene expression in acute lymphoblastic leukemia cell line (CCRF-CEM): An epigenetic perspective. J Cell Biochem, 119, 4890-6

Zadi Heydarabad M, Nikasa M, Vatanmakanian M, Azimi A, Farshdousti Hagh M (2018). Regulatory effect of resveratrol and prednisolone on MDR1 gene expression in acute lymphoblastic leukemia cell line (CCRF-CEM): An epigenetic perspective. J Cell Biochem, 119, 4890-6.

Zadi Heydarabad M, Vatanmakanian M, Abdolalizadeh J, et al (2018). Apoptotic effect of resveratrol on human T-ALL cell line CCRF-CEM is unlikely exerted through alteration of BAX and BCL2 promoter methylation. J Cell Biochem, 119, 10033-40.

Zadi Heydarabad M, Vatanmakanian M, Abdolalizadeh J, et al (2018). Apoptotic effect of resveratrol on human T-ALL cell line CCRF-CEM is unlikely exerted through alteration of BAX and BCL2 promoter methylation. J Cell Biochem, 119, 10033-40

Zaman G, Flens M, Van Leusden M, et al (1994). The human multidrug resistance-associated protein MRP is a plasma membrane drug-efflux pump. Proc Natl Acad Sci U S A, 91, 8822-6.

Zhang R, Lu M, Zhang Z, et al (2016). Resveratrol reverses P-glycoprotein-mediated multidrug resistance of U2OS/ ADR cells by suppressing the activation of the NF- $\mathrm{KB}$ and p38 MAPK signaling pathways. Oncol Lett, 12, 4147-54.

Zhang W, Murao K, Zhang X, et al (2010). Resveratrol represses YKL-40 expression in human glioma U87 cells. BMC Cancer, 10, 593.

This work is licensed under a Creative Commons AttributionNon Commercial 4.0 International License. 